\title{
Determination of the anti-polo like kinase 1 potential of novel derivatives of thiophene using oncoinformatics approach
}

\author{
Moaath Khaled Aljbreen ${ }^{1}$, Mohammed Mubarak Alrashidi', Amr Salah ${ }^{2,3}$, \\ Sibhghatulla Shaikh ${ }^{4}$, Syed Mohd Danish Rizvi ${ }^{5 *}$ \\ ${ }^{1}$ College of Pharmacy, ${ }^{2}$ Department of Pharmaceutical Chemistry, College of Pharmacy, University of Hail, Hail, Kingdom of \\ Saudi Arabia, ${ }^{3}$ National Organization for Drug Control \& Research, Cairo, Egypt, ${ }^{4}$ Department of Medical Biotechnology, \\ Yeungnam University, South Korea, ${ }^{5}$ Department of Pharmaceutics, College of Pharmacy, University of Hail, Hail, Kingdom of \\ Saudi Arabia
}

*For correspondence: Email: syedrizvi10@yahoo.com, sm.danish@uoh.edu.sa

Sent for review: 3 November 2020

Revised accepted: 23 February 2021

\begin{abstract}
Purpose: To explore the anticancer mechanistic aspect of thiophene derivatives via targeting Polo like kinase 1 (PLK1).

Methods: The PLK1 enzyme is primarily expressed in cancer cells, and blocking its active site is one of the plausible ways to target cancer. Thus, in the present study, the thiophene derivatives were tested against PLK1 by molecular docking approach.

Results: Thiophene derivatives, named $8 A, 8 B$ and 14, exhibited better interactions with PLK1 active site than the positive control, doxorubicin. Molecular docking experiments revealed that $8 A, 8 B$ and 14 interacted efficiently with PLK1, and demonstrated binding energy and inhibition constant scores of '$8.02 \mathrm{kcal} / \mathrm{mol}$ and $1.33 \mu \mathrm{M}^{\prime}$ ', '-8.65 $\mathrm{kcal} / \mathrm{mol}$ and $0.454 \mu \mathrm{M}^{\prime}$ and '-8.33 $\mathrm{kcal} / \mathrm{mol}$ and $0.788 \mu \mathrm{M}^{\prime}$ ', respectively. In contrast, doxorubicin-PLK1 interaction had binding energy of $-7.95 \mathrm{kcal} / \mathrm{mol}$ and inhibition constant of $2.75 \mu \mathrm{M}$.

Conclusion: These results predict that thiophene derivatives $8 A, 8 B$ and 14 might exert anticancer effect by inhibiting PLK1 activity. Although, wet lab experiments are required to validate the data, however, these results may pave the way for the development of novel PLK1 inhibitors for anticancer therapy.
\end{abstract}

Keywords: Anticancer therapy, Cancer cells, Molecular docking, Polo-like kinase 1, Thiophene derivatives

\begin{abstract}
This is an Open Access article that uses a fund-ing model which does not charge readers or their institutions for access and distributed under the terms of the Creative Commons Attribution License (http://creativecommons.org/licenses/by/4.0) and the Budapest Open Access Initiative (http://www.budapestopenaccessinitiative.org/read), which permit unrestricted use, distribution, and reproduction in any medium, provided the original work is properly credited.

Tropical Journal of Pharmaceutical Research is indexed by Science Citation Index (SciSearch), Scopus, International Pharmaceutical Abstract, Chemical Abstracts, Embase, Index Copernicus, EBSCO, African Index Medicus, JournalSeek, Journal Citation Reports/Science Edition, Directory of Open Access Journals (DOAJ), African Journal Online, Bioline International, Open-J-Gate and Pharmacy Abstracts
\end{abstract}

\section{INTRODUCTION}

Cancer is a major health concern globally, and a major cause of mortality in the United States [1]. Metastasis, recurrence and drug resistance are the factors that are associated with increased mortality rate. Conventional chemotherapeutic agents, such as, antimitotic and antimetabolites drugs [2] are not adequate to control the majority of cancers, and frequently direct to drug-resistant 
and metastasis [3]. Therefore, the current situation has become more challenging, as the effectiveness of existing chemotherapy treatments is considerably decreasing. This has encouraged researchers to develop new drugs that can act on emerging cancer-specific targets, for instance polo-like kinase-1 (PLK1), which can be used as monotherapy as well as in combination with existing chemotherapy, for improved cancer treatment [4].

Antimitotics have been considered as the therapeutic option for the patient's with solid tumors, and PLK1 is one of the promising antimitotic targets [5]. The first polo-like kinase family member (i.e. Polo) was identified in Drosophila melanogaster [6]. Later, four PLK family members- PLK1, PLK2, PLK3 and PLK4 were recognized [7]. Out of all PLKs, the bestcharacterized one is PLK1, which is an antimitotic target expressed exclusively in dividing cells. Expression of PLK1 fluctuates during the progression of cell cycle, and peaks at M-phase of the cell cycle [8]. PLKs have various functions during mitosis, and PLK1 activity is vital for almost every mitosis step via its various phosphorylation and protein binding activities. PLK1 phosphorylates the cyclin B1 and Cdc25C to promote the mitotic entry [9].

PLK1 has emerged as a potent target enzyme for cancer drug development. The rationale behind development of PLK1 as a cancer target includes its overexpression in cancer, association with poor prognosis and ability to cause multinucleation [10,11]. In addition, increased PLK1 expression might help the cell to override $\mathrm{G} 2$ checkpoint arrest caused by DNA damage [12]. It has been reported that constitutive expression of PLK1 could lead to alter NIH 3T3 fibroblasts cells [13] and pro-apoptotic function of p53 protein as well [14].

Since PLK1 is linked to tumorigenesis, and can be targeted by various compounds or agents, it provides a potential route for the advancement of novel anticancer treatment. Earlier, one of the author's has explored the potency of novel thiophene derivatives (compounds named as 8A, $8 B$ and 14) against various cancer cell lines, and found their potent activity against brain cancer, breast adenocarcinoma and non-small cell lung cancer cell lines [15]. However, the underlying mechanism of their anticancer effects still needs to be elucidated.

In continuance, to explore the mechanistic aspects, we predicted the potential of thiophene derivatives against cancer specific target, i.e., PLK1 in the present study.

\section{EXPERIMENTAL}

\section{Ligand and protein preparations}

The 3D structure of PLK1 (PDB ID: 2OWB) was retrieved from the Protein Data Bank. ChemDraw was used to design the structure of thiophene derivatives named as $8 \mathrm{~A}, 8 \mathrm{~B}$ and 14 that had been synthesized and tested against cancer cell lines by Dr. Amr S. Abouzied [15].

\section{Calculation of physicochemical properties and toxicity risk prediction}

Molinspiration property calculation tool (http://www.molinspiration.com/cgi-

bin/properties) was employed to check the physicochemical properties of thiophene derivatives compounds. Various parameters such as topological polar surface area (TPSA), molecular weight, miLogP, the number of hydrogen bond donors, number of hydrogen bond acceptors, number of rotatable bonds, and violations of Lipinski's rule of five [16] were estimated using Molinspiration tool.

Further, Absorption (B) was calculated using Eq 1 [17]:

$E(W)=128-(0,843 \times T P S A) \ldots \ldots(1)$

Orisis datawarrior tool was used to predict the toxicity potential of thiophene derivatives. The prediction is based on relative analysis of thiophene derivatives with the pre-calculated set of previously explored structural molecules present in databases.

\section{Molecular docking}

The thiophene derivatives (compound $8 \mathrm{~A}, 8 \mathrm{~B}$ and 14) and control were docked with the PLK1 catalytic domain individually with Auto dock 4.2 tools following the method of Rizvi et al [18]. To target catalytic site of PLK1 enzyme, $x, y$, and $z$ coordinate values were used as $0.069,23.58$ and 66.741 , respectively, and Grid box dimension was set as $40 \AA \times 40 \AA \times 40 \AA$ with a spacing of $0.375 \AA$. The figures were produced using Discovery Studio 2.5 (Accelrys).

\section{Ligplot analysis}

To determine the hydrogen and hydrophobic interactions between important amino acid residues of PLK1 with thiophene derivatives the 'thiophene derivatives-PLK1'complexes were analyzed by LIGPLOT ${ }^{+}$Version v.2.1. 


\section{RESULTS}

\section{Physicochemical properties prediction}

The physicochemical and drug-likeness properties of the thiophene derivatives (compounds 8A, 8B, and 14) was performed by Molinspiration property calculation tool. It was found that compounds $8 \mathrm{~A}, 8 \mathrm{~B}$, and 14 were under the defined parameters of molecular weight (<500Da), hydrogen bond acceptor $(\leq 10)$, and hydrogen bond donor $(\leq 5)$ (Table 1 ) and none of the compounds violated the Lipinski's rule of five. The orisis software predicted the toxicity in the form of mutagenicity, tumorigenicity, irritability and reproductive effects (Table 2). Out of all the compounds tested, only compound 14 and doxorubicin predicted to have the irritability and reproductive effect, respectively.

\section{Molecular docking and Ligplot analysis}

Leu59, Gly60, Lys61, Cys67, Ala80, Leu130, Glu131, Leu132, Cys133, Arg134, Arg136, and Phe183 residues of PLK1 was found to interact with compound 8A (Figure 1a); while compound 8B was found to interact with Leu59, Gly60, Lys61, Gly62, Ala65, Lys66, Cys67, Ala80, Lys82, Val114, Glu131, Leu132, Cys133, Arg134, Arg136, Phe183, and Asp194 residues of PLK1 (Figure 2a). In addition, compound 14 was found to interact with Leu59, Gly60, Lys61 Gly62, Ala65, Cys67, Ala80, Val114, Leu130, Glu131, Leu132, Cys133, Arg134, Arg135, Arg136, and Phe183 residues of PLK1 (Figure 3a).

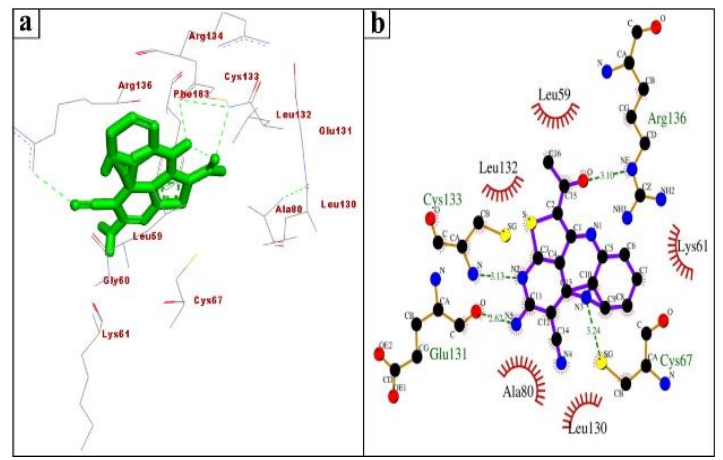

Figure 1: a) Molecular interaction of compound $8 \mathrm{~A}$ (stick representation) with PLK1. b) Ligplot analysis of PLK1 showing the hydrogen bond (green dash line) and hydrophobic interaction (red arcs) with compound $8 \mathrm{~A}$

The free binding energy $(\Delta G)$ for compound $8 A-$ PLK1, compound 8B-PLK1, and compound 14PLK1 catalytic domain interactions were -8.02, 8.65 , and $-8.33 \mathrm{kcal} / \mathrm{mol}$, respectively, while the inhibition constant (ki) values were 1.33, 0.454, and $0.788 \mu \mathrm{M}$, respectively (Table 3 ).

Table 1: Physicochemical parameters for the compounds

\begin{tabular}{|c|c|c|c|c|c|c|c|c|c|}
\hline \multirow{2}{*}{\multicolumn{2}{|c|}{ S. No. Compound Name }} & \multicolumn{8}{|c|}{ Physiochemical parameters } \\
\hline & & 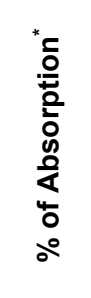 & 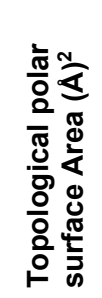 & $\begin{array}{l}\frac{ \pm}{0} \\
\frac{0}{0} \\
\frac{0}{3} \\
\frac{\pi}{5} \\
\frac{0}{J} \\
\frac{d}{0} \\
\sum\end{array}$ & 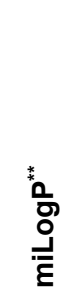 & 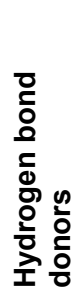 & 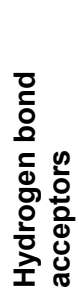 & 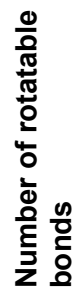 & 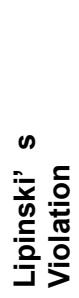 \\
\hline & Rule & - & - & $<500$ & $\leq 5$ & $<5$ & $<10$ & $\leq 10$ & $\leq 1$ \\
\hline$\overline{1}$ & Compound $8 \mathrm{~A}$ & 68.34 & 117.83 & 323.38 & 2.98 & 5 & 6 & 3 & 0 \\
\hline 2 & Compound 8B & 67.48 & 120.34 & 370.43 & 3.44 & 5 & 7 & 6 & 0 \\
\hline 3 & Compound 14 & 69.29 & 115.10 & 368.42 & -4.55 & 2 & 7 & 6 & 0 \\
\hline 4 & Doxorubicin*** & 35.72 & 212.39 & 545.54 & 0.62 & 9 & 12 & 5 & 3 \\
\hline
\end{tabular}

${ }^{*}$ Absorption was calculated as per Eq 1; ${ }^{* *}$ miLogP is the logarithm of compound partition coefficient between $n$ octanol and water. ${ }^{* * *}$ Doxorubicin is the anticancer control drug used for comparative analysis

Table 2: Toxicity risk assessment of compounds

\begin{tabular}{lcccc}
\hline Compound & \multicolumn{4}{c}{ Toxicity risk } \\
\cline { 2 - 5 } & Mutagenity & Tumorigenicity & Irritability & Reproductive effect \\
\hline Compound 8A & None & None & None & None \\
Compound 8B & None & None & None & None \\
Compound 14 & None & None & High & None \\
Doxorubicin* & None & None & None & High \\
\hline
\end{tabular}




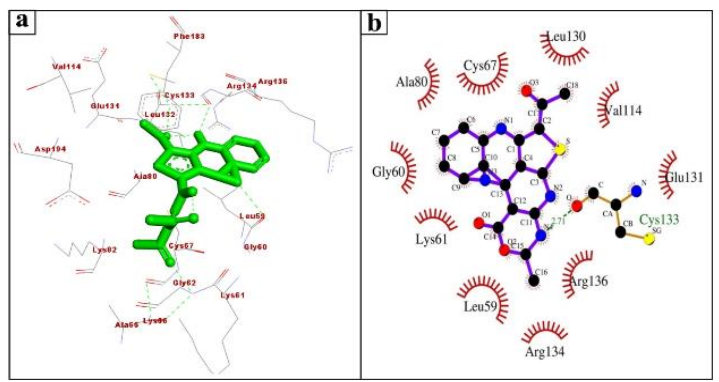

Figure 2: (a) Molecular interaction of compound 8B (stick representation) with PLK1. (b) Ligplot analysis of PLK1 showing the hydrogen bond (green dash line) and hydrophobic interaction (red arcs) with compound $8 \mathrm{~B}$

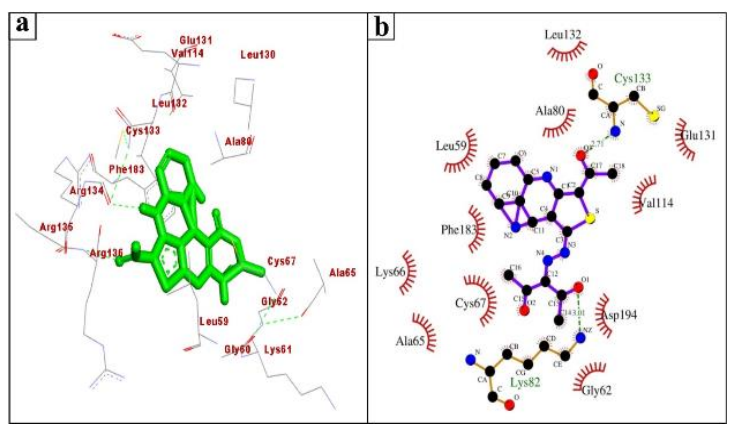

Figure 3: (a) Molecular interaction of compound 14 (stick representation) with PLK1. (b) Ligplot analysis of PLK1 showing the hydrogen bond (green dash line) and hydrophobic interaction (red arcs) with compound 14.

The amino acid residues Cys67, Glu131, Cys133, and Arg136 of PLK1 made hydrogen bond with compound $8 \mathrm{~A}$, while Cys 133 was the common $\mathrm{H}$-bond interacting amino acid residue of PLK1 with compound 8A, 8B, and 14 (Figure $1 b, 2 b$, and $3 b)$.

Doxorubicin is a chemotherapeutic agent in the treatment of different kinds of cancer, and in this study, it was used as the control compound. Leu59, Gly60, Lys61, Cys67, Ala80, Glu131, Leu132, Cys133, Arg134, Arg136, and Phe183 were the common interacting amino acid residues of PLK1 with doxorubicin, compounds $8 \mathrm{~A}, 8 \mathrm{~B}$ and 14 (Figures $1-3$ and Figure 4). The $\triangle G$ and $\mathrm{ki}$ values for doxorubicin-PLK1 interaction were $-7.95 \mathrm{kcal} / \mathrm{mol}$ and $2.75 \mu \mathrm{M}$, respectively (Table 3 ).

\section{DISCUSSION}

In the past few decades, promising anticancer compounds fails at different clinical trials stages as a result of inappropriate pharmacokinetic profiling. However, to reduce the failure rate, physicochemical profiling of new drug candidates has become an important part of drug discovery at present. These analyses involve assessment of various molecular descriptors that would help in prediction of drug-likeliness. There are various in silico tools available for calculating the molecular properties of a compound in order to assess its pharmacokinetic profile. In fact, these in silico tools have helped to overcome the issues related to failure of clinical trials [19].

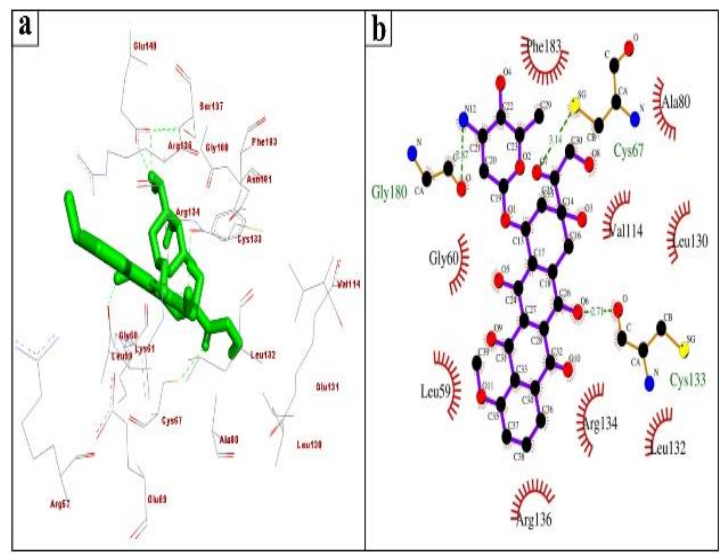

Figure 4: (a) Molecular interaction of Doxorubicin (stick representation) with PLK1. (b) Ligplot analysis of PLK1 showing the hydrogen bond (green dash line) and hydrophobic interaction (red arcs) with doxorubicin

Table 3: Binding energy of compounds with PLK1

\begin{tabular}{lcc}
\hline Drug/ligand & $\begin{array}{c}\text { Free binding } \\
\text { energy } \\
\text { (kcal/mol) }\end{array}$ & $\begin{array}{c}\text { Inhibition } \\
\text { constant }(\boldsymbol{\mu M})\end{array}$ \\
\hline Compound 8A & -8.02 & 1.33 \\
Compound 8B & -8.65 & 0.454 \\
Compound 14 & -8.33 & 0.788 \\
Doxorubicin* & -7.95 & 2.75 \\
\hline \multicolumn{2}{l}{${ }^{*}$ Doxorubicin is the anticancer control drug used for } \\
comparative analysis
\end{tabular}

Molinspiration tool was used in this study to screen the thiophene derivatives (compounds $8 \mathrm{~A}, 8 \mathrm{~B}$, and 14) as drug candidates based on Lipinski's Rule of Five (Table 1). Lipinski's Rule of Five is based on the observation that the majority of oral drugs have a molecular weight $\leq 500$, Log $\mathrm{P}$ value (logarithmic value of partition coefficient between $n$-octanol and water) $\leq 5$, hydrogen bond donor sites $<5$, hydrogen bond acceptor sites $<10$ and rotatable bonds $\leq 10$. Any compound which violates more than one of these rules has poor bioavailability. In this study, none of the tested compounds showed violation of Lipinski's Rule of Five except positive control doxorubicin. Thus, these compounds could be considered as potential drug candidates.

PLK1 is over-expressed in various cancer cells, 
including breast cancer cells. Interestingly, PLK1 inhibition restrains cancer cells growth without affecting normal cells [20]. Therefore, it is regarded as an important therapeutic target in the management of various cancers. In this study, thiophene derivatives $8 \mathrm{~A}, 8 \mathrm{~B}$ and 14 strongly bound to the PLK1 catalytic site. Leu59, Gly60, Lys61, Cys67, Ala80, Glu131, Leu132, Cys133, Arg134, Arg136, and Phe183 were the common interacting residues of PLK1 catalytic site with the compound $8 \mathrm{~A}, 8 \mathrm{~B}$, and 14 . Interestingly, these residues of PLK1 enzyme have been reported to interact with the FDA approved anti-cancer drugs [21].

Amino acid residues, Leu59, Ala80, Val114, Leu132 and Cys133 of PLK1 have been reported to make hydrophobic interaction with natural compound (1,4-cyclohexylphenyl) ethanone [22]. Consistent with this, Leu59 and Ala80 residues of PLK1 were commonly involved in hydrophobic interaction with compound 8A, 8B and 14 (Figure $1 \mathrm{~b}, 2 \mathrm{~b}$, and $3 \mathrm{~b}$ ). Moreover, both hydrogen bonds and hydrophobic interactions plays a vital role in the binding stability of ligand to its corresponding target protein [23].

The strength of binding between a ligand and protein is determined in terms of binding energy, and a higher (negative) energy represents the efficient binding of a ligand to the target protein or receptor [24]. Interestingly, this study shows that thiophene derivatives (compounds $8 \mathrm{~A}, 8 \mathrm{~B}$, and 14) have better binding with PLK1 than the control drug (doxorubicin) in terms of the binding energy (Table 3), suggesting that these compounds might exert their anticancer effect by inhibiting PLK1 enzyme.

\section{CONCLUSION}

The molecular docking results predict that all the thiophene derivatives (compounds 8A, 8B, and 14) have better binding affinity towards PLK1 enzyme than the control drug (doxorubicin) with respect to $\mathrm{Ki}$ and $\Delta \mathrm{G}$ values. Thus, the findings provide a predictive mechanistic insight into the mode of anticancer action of these thiophene derivatives, and might aid the further investigation of the exact mechanistic aspects of the anticancer potentials of the thiophene derivatives.

\section{DECLARATIONS}

\section{Conflict of interest}

No conflict of interest is associated with this work.

\section{Contribution of authors}

We declare that this work was done by the authors named in this article and all liabilities pertaining to claims relating to the content of this article will be borne by the authors.

\section{Open Access}

This is an Open Access article that uses a funding model which does not charge readers or their institutions for access and distributed under the terms of the Creative Commons Attribution License (http://creativecommons.org/licenses/by/ 4.0) and the Budapest Open Access Initiative (http://www.budapestopenaccessinitiative.org/rea d), which permit unrestricted use, distribution, and reproduction in any medium, provided the original work is properly credited.

\section{REFERENCES}

1. Siegel RL, Miller KD, Jemal A. Cancer statistics 2016. CA Cancer J Clin 2016; 66: 7-30.

2. Gerber DE. Targeted therapies: a new generation of cancer treatments. Am Fam Physician 2008; 77: 311319.

3. Nastiuk KL, Krolewski JJ. Opportunities and challenges in combination gene cancer therapy. Adv Drug Deliv Rev 2016; 98: 35-40.

4. Gutteridge REA, Ndiaye MA, Liu X, Ahmad N. Plk1 inhibitors in cancer therapy: from laboratory to clinics. Mol Cancer Ther 2016; 15: 1427-1435.

5. Pitts TM, Davis SL, Eckhardt SG, Bradshaw-Pierce EL. Targeting nuclear kinases in cancer: development of cell cycle kinase inhibitors. Pharmacol Ther 2014; 142: 258269.

6. Fenton B, Glover DM. A conserved mitotic kinase active at late anaphase-telophase in syncytial Drosophila embryos. Nature 1993; 363: 637-640.

7. de Cárcer G, Manning G, Malumbres M. From Plk1 to Plk5: functional evolution of polo-like kinases. Cell Cycle 2011; 10: 2255-2262.

8. Lake RJ, Jelinek WR. Cell cycle- and terminal differentiation-associated regulation of the mouse mRNA encoding a conserved mitotic protein kinase. Mol Cell Biol 1993; 13: 7793-7801.

9. Toyoshima-Morimoto F, Taniguchi E, Nishida E. Plk1 promotes nuclear translocation of human Cdc25C during prophase. EMBO Rep 2002; 3: 341-348.

10. Eckerdt F, Yuan J, Strebhardt K. Polo-like kinases and oncogenesis. Oncogene 2005; 24: 267-276.

11. Jang YJ, Lin CY, Ma S, Erikson RL. Functional studies on the role of the C-terminal domain of mammalian pololike kinase. Proc Natl Acad Sci USA 2002; 99: 19841989. 
12. van Vugt MA, Bras A, Medema RH. Polo-like kinase-1 controls recovery from a G2 DNA damage induced arrest in mammalian cells. Mol Cell 2004; 15: 799-811.

13. Smith MR, Wilson ML, Hamanaka $R$, Chase $D$, Kung $H$, Longo DL, Ferris DK. Malignant transformation of mammalian cells initiated by constitutive expression of the polo-like kinase. Biochem Biophys Res Commun 1997; 234: 397-405.

14. Ando K, Ozaki T, Yamamoto H, Furuya K, Hosoda M, Hayashi S, Fukuzawa M, Nakagawara A. Polo-like kinase 1 (Plk1) inhibits p53 function by physical interaction and phosphorylation. J Biol Chem 2004; 279 : 25549-25561.

15. Mohareb RM, Sherif SM, Wardakhan WW, Abouzied AS. New approaches for the use of acetoacetanilide in the Synthesis of thiophenes and their fused derivatives with anti-tumor activity. Open Access Library Journal 2014; 1: 1-16.

16. Lipinski CA, Lombardo F, Dominy BW, Feeney PJ. Experimental and computational approaches to estimate solubility and permeability in drug discovery and development settings. Adv Drug Deliv Rev 2001; 46: 326.

17. Zhao YH, Abraham MH, Le J, Hersey A, Luscombe CN, Beck G, Sherborne B, Cooper I. Rate-limited steps of human oral absorption and QSAR studies. Pharm Res 2002; 19: 1446-1457.

18. Rizvi SM, Shakil S, Haneef M. A simple click-by-click protocol to perform docking: AutoDock 4.2 made easy for non-bioinformaticians. EXCLI J 2013; 12: 831-857.

19. Azam F, Mohamed N, Alhussen F. Molecular interaction studies of green tea catechins as multitarget drug candidates for the treatment of Parkinson's disease: computational and structural insights. Network: Computation in neural systems 2015; 26: 97-115.
20. Maire V, Némati $F$, Richardson $M$, Vincent-Salomon A, Tesson B, Rigaill G, Gravier E, Marty-Prouvost B, De Koning L, Lang G, Gentien D, Dumont A, Barillot $E$, Marangoni E, Decaudin D, Roman-Roman S, Pierré A, Cruzalegui F, Depil S, Tucker GC, Dubois T. Polo-like kinase 1: a potential therapeutic option in combination with conventional chemotherapy for the management of patients with triple-negative breast cancer. Cancer Res 2013; 73: 813-823.

21. Rizvi SMD, Alshammari AAA, Almawkaa WA, Ahmed ABF, Katamesh A, Alafnan A, Almutairi TJ, Alshammari $R F$. An oncoinformatics study to predict the inhibitory potential of recent FDA-approved anti-cancer drugs against human Polo-like kinase 1 enzyme: a step towards dual-target cancer medication. 3 Biotech 2019; 9: 70.

22. Rizvi SMD, Shakil S, Zeeshan M, Khan MS, Shaikh S, Biswas D, Ahmad A, Kamal MA. An enzoinformatics study targeting polo-like kinases-1 enzyme: Comparative assessment of anticancer potential of compounds isolated from leaves of Ageratum houstonianum. Phcog Mag 2014; 10: 14-21.

23. Shaikh S, Rizvi SMD, Hameed N, Biswas D, Khan M, Shakil S, Kamal MA. Aptiom (Eslicarbazepine acetate) as a dual inhibitor of $\beta$-secretase and Voltage-gated sodium channel: advancement in Alzheimer's diseaseEpilepsy linkage via an enzoinformatics study. CNS \& Neurol Dis - Drug Tar 2014; 13: 1258-1262.

24. Shaikh S, Rizvi SM, Suhail T, Shakil S, Abuzenadah AM, Anis $R$, Naaz $D$, Haneef $M$, Ahmad A, Choudhary $L$. Prediction of anti-diabetic drugs as dual inhibitors against acetylcholinesterase and beta-secretase: a neuroinformatics study. CNS \& Neurol Dis - Drug Tar 2016; 15: 1216-1221. 